\title{
The Von Kármán constant retrieved from CASES-97 dataset using a variational method
}

\author{
Y. Zhang ${ }^{1}$, J. $^{\mathrm{Ma}^{2}}$, and Z. $\mathrm{Cao}^{3}$ \\ ${ }^{1}$ Zhuhai Air Traffic Management Station CAAC, Zhuhai International Airport, Sanzao, Zhuhai 519040, \\ Guangdong, P. R. China \\ ${ }^{2}$ Air Quality Research Division, Science and Technology Branch, Environment Canada, 4905 Dufferin Street, \\ Toronto, Ontario M3H 5T4, Canada \\ ${ }^{3}$ Meteorological Service of Canada, 4905 Dufferin Street, Toronto, Ontario M3H 5T4, Canada
}

Received: 4 March 2008 - Published in Atmos. Chem. Phys. Discuss.: 17 July 2008

Revised: 19 September 2008 - Accepted: 2 November 2008 - Published: 5 December 2008

\begin{abstract}
A variational method is developed to retrieve the von Kármán constant $\kappa$ from the CASES-97 dataset, collected near Wichita, Kansas, the United States from 6 April to 24 May 1997. In the variational method, a cost function is defined to measure the difference between observed and computed gradients of wind speed, air temperature and specific humidity. An optimal estimated von Kármán constant is obtained by minimizing the cost function through adjusting values of the von Kármán constant. Under neutral stratification, the variational analysis confirms the conventional value of $\kappa(=0.40)$. For non-neutral stratification, however, $\kappa$ varies with stability. The computational results show that the $\kappa$ decreases monotonously from stable to unstable stratification. The variational calculated mean value of the von Kármán constant is $0.383 \sim 0.390$ when the atmospheric stratification is taken into consideration. Relations between $\kappa$ and surface momentum and heat flux are also examined.
\end{abstract}

\section{Introduction}

As a well-known scaling factor, the von Kármán constant $\kappa$ was introduced over 60 years ago to scale the logarithmic law of mean wind profile in the atmospheric surface boundarylayer. The logarithmic velocity law has been verified in the atmosphere surface layer when a true neutral condition occurs (Garratt, 1992). The von Kármán constant $\kappa$ has been

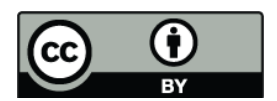

Correspondence to: J. Ma

(Jianmin.ma@ec.gc.ca) assumed to be a universal constant under the neutral atmospheric condition. Extensive wind-tunnel experimental data has revealed that $\kappa=0.4$. However, uncertainties of the von Kármán constant seem to exist under a realistic atmospheric condition. These uncertainties led to continuous debating on the true value of the von Kármán constant. Suggested values of the von Kármán constant are ranging from 0.35 (Businger et al., 1971) to 0.46 (Sheppard, 1947). Until recent years, $\kappa$ values and factors affecting the von Kármán constant have been still pursued in the meteorological community. Frenzen and Vogel (1995) find that $\kappa$ is actually a weak variable that decreases with increasing in the roughness Reynolds number, and therefore weakly depends on the surface roughness length. They suggest that $\kappa=0.39 \pm 1 \%$. Their finding seems, however, not to gain a wide support from other measurements. Using Parlange and Katul's (1995) measurement data, Högström (1996) raises an objection to Frenzen and Vogel's (1995) hypothesis that $\kappa$ is dependent on the roughness Reynolds number. He concludes that $\kappa$ is indeed a constant. Cai and Steyn (1996) investigate the von Kármán constant using a large eddy simulation technique and find that $\kappa$ is dependent on "Smagororinsky-Model Reynolds number", a ratio of advection term to sub-grid scale diffusion term in the Navier-Stokes equation. They conclude that $\kappa$ is unlikely larger than 0.4. A most recent review and evaluation on the von Kármán constant is given by Andreas et al. (2006). From their calculation using two large atmospheric data sets, Andreas et al. (2006) suggest that $\kappa$ is a constant at $0.387 \pm 0.003$ for a broad range of roughness Reynolds number $2 \leq R_{e} \leq 100$. 
A number of reasons have been highlighted to explain why the lack of precise in determination of the von Kármán constant in the atmosphere. Strictly speaking, since wind speed, air temperature and humidity in the surface boundary-layer can be described by the logarithmic laws which are scaled by the von Kármán constant, the estimate of the von Kármán constant must be carried out with accurate measurements of wind speed, air temperature and humidity as well as their gradients under neutral conditions. In reality, this requirement is seldom satisfied. Adoption of different measurement techniques may result in different values of the von Kármán constant (e.g., Kondo and Sato, 1982). Wind sensors widely used in the field studies of the boundary - layer meteorology may lead to systematic errors. The limitations of data quantity may affect the quality of the evaluation of the von Kármán constant (Zhang et al., 1988) as well. Stability effects must be also taken into account in the estimate of $\kappa$. Frenzen and Vogel (1995) indicate that measurements made in weak stable stratifications can lead to incorrect estimates of $\kappa$ because the flow would become decoupled from the surface, a phenomenon often occurring over smooth surfaces where there exists a weak mechanical mixing. They have claimed that the remaining uncertainty on the atmospheric value of $\kappa$ in the mid 1990s became small and the experimental estimates of $\kappa$ lies in the range from 0.36 to 0.40 . In other words, $\kappa$ value is smaller than 0.4 . However, the stability effects on the estimate of $\kappa$ using the classical Monin-Obukhov similarity relationships are still not very clear. In addition, the usual method to compute the von Kármán constant (e.g., so called " $\phi_{m}$ " method, Zhang et al., 1988) appears not to consider sufficiently the measurement errors. " $\phi_{m}$ " method derives the $\kappa$ value from a number of wind profile measurements by fitting an empirical curve to non-dimensional measures of the wind shear. Hence, the result from " $\phi_{m}$ " method depends on how uniformly the estimated wind shear values happen to be distributed along the fitting curve, and on the accuracy of measurements (Frenzen and Vogel, 1995). Furthermore, the logarithmic law for mean velocity profiles has been extended to mean air temperature, humidity and other scalars, though few reports have been documented to investigate the von Kármán constant when it is used to scale the logarithmic laws of air temperature and other scalars. These comments suggest that some uncertainties still remain in the determination of $\kappa$. These uncertainties are likely to affect considerably the accurate estimate of momentum, sensible heat, humidity and concentration fluxes, because these fluxes are proportional to $\kappa^{2}$ (Fleagle and Businger, 1980). It is, therefore, interesting to further investigate those uncertainties and factors that may affect $\kappa$ value.

With up-to-date measurement techniques, a number of field experiments in the atmospheric boundary - layer have been conducted in recent years. The large quantity of measurement data from these filed experiments provides a new insight into the reevaluation of the von Kármán constant. In this study, the CASES-97 (Surface Qualls Surface Flux
Site) dataset is used to estimate the von Kármán constant. The dataset includes wind speeds, air temperature and humidity profile data at a 10 min interval with 6628 time levels which were measured at a relatively flat, grass covered site (see next section for details). To apply effectively this large amount of data to calculate the von Kármán constant, a variational algorithm is developed. The variational method provides a conceptually simple and self-consistent procedure to fully and simultaneously use the information provided by wind, air temperature and humidity profiles, as well as other variables measured in the atmospheric surface layer (Ma and Daggupaty, 1999; Cao and Ma, 2005; Cao et al., 2006).

The paper is organized as follows. The CASES-97 data are described in Sect. 2. Physical constraints and variational method to determine $\kappa$ are presented in Sect. 3. In Sect. 4, the computational results are presented and discussed. Conclusions are given in Sect. 5.

\section{Data}

CASES-97 (Surface Qualls Surface Flux Site) dataset is a processed dataset from the Qualls surface flux site near Wichita, Kansas, the United States $\left(37.738^{\circ} \mathrm{N}, 97.184^{\circ} \mathrm{W}\right)$. The site is relatively flat and covered with short grass. Predominant wind directions were from the N-NW and from the SW-S-SE. The field is approximately rectangular with $400 \mathrm{~m}$ clear fetches to the north and south. The fetches to the east and west are $200 \mathrm{~m}$. The field campaign was conducted during 6 April-24 May 1997 with a time interval of $10 \mathrm{~min}$, except for a data missing period from 22:00 GMT, 1 May to 14:20 GMT, 3 May. There are total 6628 time levels of observations. Multiple level measurements of horizontal wind speed, air temperature and relative humidity were made in CASES-97 (Qualls Site). The wind speeds $\mathrm{u}\left(\mathrm{m} \mathrm{s}^{-1}\right)$ were measured at 1.0, 2.0 and $3.0 \mathrm{~m}$ above the ground surface using RM Young cup anemometers; air temperature $t\left({ }^{\circ} \mathrm{C}\right)$ and relative humidity were measured at 1 and $2 \mathrm{~m}$ above the surface using radiation and energy balance systems (REBS THP, shielded and aspirated). The dataset also includes sensible heat flux, net radiation, and ground heat flux. The sensible heat flux $H\left(\mathrm{~W} \mathrm{~m}^{-2}\right)$ was measured with the eddy correlation technique at $10 \mathrm{~Hz}$ using CSI (Campbell Scientific Inc.) 1-D sonic anemometer, and the latent heat flux $\operatorname{LE}\left(\mathrm{W} \mathrm{m}^{-2}\right)$ was computed from the energy budget method. In the present study, to avoid the uncertainty due to the effects of the roughness lengths on evaluation of the von Kármán constant (Frenzen and Vogel, 1995), wind speed, air temperature and relative humidity data at 1 and $2 \mathrm{~m}$ height are used. Relative humidity data are converted to the specific humidity in order to apply the similarity relationship of humidity in the subsequent variational analysis. Because in the present study we used the information of wind, air temperature and humidity at the two measurement levels ( 1 and $2 \mathrm{~m}$ ), the roughness 
length for momentum, heat and humidity as well as the zerodisplacement height were not taken into account.

\section{Logarithmic profiles and variational method}

The objective of the variational algorithm in this study is to find an optimal estimate of the von Kármán constant through minimizing a cost function. The cost function defined in the variational method is introduced to measure the difference between computed and observed gradients of the wind speed, temperature and humidity (e.g., Cao et al., 2006)

$$
\begin{aligned}
& J=\frac{1}{2}\left[W_{u}\left(\Delta u-\Delta u^{\mathrm{obs}}\right)^{2}+W_{T}\left(\Delta T-\Delta T^{\mathrm{obs}}\right)^{2}\right. \\
& \left.+W_{q}\left(\Delta q-\Delta q^{\mathrm{obs}}\right)^{2}\right]
\end{aligned}
$$

where $W_{u}, W_{T}$ and $W_{q}$ are dimensional weights, the superscript "obs" stands for observation, $\Delta u=u_{2}-u_{1}$, $\Delta T=T_{2}-T_{1}, \Delta q=q_{2}-q_{1}$, the subscripts 1 and 2 stand for the heights of the first $(1 \mathrm{~m})$ and second measurement level ( $2 \mathrm{~m}$ ) above the ground, respectively. $\Delta u, \Delta T$ and $\Delta q$ are computed using the Monin-Obukhov similarity relationships (Yaglom, 1977):

$$
\begin{aligned}
& \Delta u=\frac{u_{*}}{\kappa}\left[\ln \left(\frac{z_{2}}{z_{1}}\right)-\psi_{m}\left(\frac{z_{2}}{L}\right)+\psi_{m}\left(\frac{z_{1}}{L}\right)\right], \\
& \Delta T=\frac{T_{*}}{\kappa}\left[\ln \left(\frac{z_{2}}{z_{1}}\right)-y_{h}\left(\frac{z_{2}}{L}\right)+y_{h}\left(\frac{z_{1}}{L}\right)\right],
\end{aligned}
$$

and

$$
\Delta q=\frac{q_{*}}{\kappa}\left[\ln \left(\frac{z_{2}}{z_{1}}\right)-y_{q}\left(\frac{z_{2}}{L}\right)+y_{q}\left(\frac{z_{1}}{L}\right)\right],
$$

where $z_{1}=1 \mathrm{~m}$ and $z_{2}=2 \mathrm{~m}, u_{*}$ is the friction velocity, $T_{*}$ and $q_{*}$ are the turbulent surface temperature and specific humidity scales, respectively. Obukhov length $L=u_{*}^{2} \bar{T} /\left(\kappa g T_{*}\right)$. $\psi_{m}, \psi_{h}$ and $\psi_{q}$ in Eqs. (2)-(4) are universal similarity functions for wind speed, temperature and humidity profiles, respectively (e.g., Businger et al., 1971):

$$
\begin{aligned}
& \psi_{m}=2 \ln \left(\frac{1+x}{2}\right)+\ln \left(\frac{1+x^{2}}{2}\right)-2 \tan ^{-1}(x)+\frac{\pi}{2}, \\
& y_{h}=2 \ln \left(\frac{1+y}{2}\right), \quad y_{q}=y_{h},
\end{aligned}
$$

for unstable conditions $(z / L<0)$, where

$x=\left[1-\gamma_{m}\left(\frac{z}{L}\right)\right]^{1 / 4}$,

and

$y=\left[1-\gamma_{h}\left(\frac{z}{L}\right)\right]^{1 / 2} ;$

and $y_{m}=-\beta_{m} \frac{z}{L}$

$y_{h}=-\beta_{h} \frac{z}{L}$

for stable conditions $(z / L>0)$. The constants in the above formulae are expected to be sensitive to the von Kármán constant. Frenzen and Vogel (1995) find that the increase in $\gamma_{m}$ by reducing $\kappa$ by $5 \%$ decreases the wind gradient function $\phi_{m}$ by $8 \%$ at $z / L=-0.1$ and by $11 \%$ at $z / L=-1.0$. In the first instance, in the present study we shall use those values given by Businger et al. (1971) at $\left(\gamma_{m}, \gamma_{h}, \beta_{m}, \beta_{h}\right)=(15,9$, $4.7,6.35)$. Another group of the constants will be also used to test the sensitivity of $\kappa$ to these constants (see Sect. 4).

The cost function, as shown in Eq. (1), contains complete information of wind, air temperature and humidity profiles in the surface boundary-layer. The minimum points of the cost function $J$ give the optimal estimates of $\kappa$. At these points, the gradient of $J$ with respect to $\kappa$ tends to be zero:

$\frac{\partial J}{\partial k}=0$.

This gradient can be expressed as,

$$
\begin{aligned}
& \frac{\partial J}{\partial k}=W_{u}\left(\Delta u-\Delta u^{\mathrm{obs}}\right) \frac{\partial \Delta u}{\partial k}+W_{T}\left(\Delta T-\Delta T^{\mathrm{obs}}\right) \\
& \frac{\partial \Delta T}{\partial k}+W_{q}\left(\Delta q-\Delta q^{\mathrm{obs}}\right) \frac{\partial \Delta q}{\partial k},
\end{aligned}
$$

The derivatives of the wind speed, air temperature and specific humidity with respect to $\kappa$ can be derived from Eqs. (2)(7) using the chain rule of differentiation,

$$
\begin{aligned}
& \frac{\partial \Delta u}{\partial k}=\left\{\begin{array}{l}
-\frac{u_{*}}{\kappa^{2}} \ln \left(\frac{z_{2}}{z_{1}}\right) L>0, \\
-\frac{\Delta u}{\kappa}+\frac{u_{*}}{\kappa}\left[-\frac{\partial \psi_{m}\left(z_{2} / L\right)}{\partial k}+\frac{\partial \psi_{m}\left(z_{1} / L\right)}{\partial k}\right] \quad L \leq 0,
\end{array},\right. \\
& \frac{\partial \Delta T}{\partial k}=\left\{\begin{array}{l}
-\frac{T_{*}}{\kappa^{2}} \ln \left(\frac{z_{2}}{z_{1}}\right), \quad L>0 \\
-\frac{\Delta T}{k}+\frac{T_{*}}{k}\left[-\frac{\partial \psi_{h}\left(z_{2} / L\right)}{\partial k}+\frac{\partial \psi_{h}\left(z_{1} / L\right)}{\partial k}\right], L \leq 0
\end{array},\right.
\end{aligned}
$$

and

$$
\frac{\partial \Delta q}{\partial k}=\left\{\begin{array}{l}
-\frac{q_{*}}{\kappa^{2}} \ln \left(\frac{z_{2}}{z_{1}}\right), \quad L>0 \\
-\frac{\Delta q}{k}+\frac{q_{*}}{k}\left[-\frac{\partial \psi_{h}\left(z_{2} / L\right)}{\partial k}+\frac{\partial \psi_{h}\left(z_{1} / L\right)}{\partial k}\right], L \leq 0
\end{array}\right.
$$

The derivatives of $\psi_{m}$ and $\psi_{h}$ with respect to $\kappa$ for unstable conditions in Eqs. (10)-(12) at $z_{1}$ are

$$
\frac{\partial \psi_{m}\left(z_{1} / L\right)}{\partial k}=-\frac{\gamma_{m} z_{1}}{2 \kappa L x_{1}^{3}}\left(\frac{1}{1+x_{1}}+\frac{x_{1}-1}{1+x_{1}^{2}}\right)
$$

and

$\frac{\partial \psi_{h}\left(z_{1} / L\right)}{\partial k}=-\frac{\gamma_{h} z_{1}}{\kappa L\left(1+y_{1}\right) y_{1}}$, 
Table 1. Values of the weights for wind speed, temperature and specific humidity in the cost function for seven variational experiments. Averaged von Kármán constant calculated using the variational method under stable and unstable conditions in different experiments is also listed.

\begin{tabular}{llllll}
\hline Run No & $\begin{array}{l}W_{u} \\
\left(\mathrm{~m}^{-2} \mathrm{~s}^{2}\right)\end{array}$ & $\begin{array}{l}W_{T} \\
\left(\mathrm{~K}^{-2}\right)\end{array}$ & $W_{q}$ & $\begin{array}{l}\bar{\kappa} \\
\text { Stable }\end{array}$ & $\begin{array}{l}\bar{\kappa} \\
\text { Unstable }\end{array}$ \\
\hline 1 & 10 & 100 & $10^{6}$ & $0.420(771)$ & $0.378(1767)$ \\
2 & 10 & 0 & 0 & $0.419(792)$ & $0.378(1884)$ \\
3 & 0 & 100 & 0 & $0.420(743)$ & $0.378(1736)$ \\
4 & 0 & 0 & $10^{6}$ & $0.420(1168)$ & $0.378(1690)$ \\
5 & 0 & 100 & $10^{6}$ & $0.421(749)$ & $0.378(1741)$ \\
6 & 10 & 100 & 0 & $0.420(772)$ & $0.378(1767)$ \\
7 & 10 & 0 & $10^{6}$ & $0.420(890)$ & $0.378(1881)$ \\
\hline
\end{tabular}

* values in brackets are number of samples in stable and unstable. conditions.

where $x_{1}$ and $y_{1}$ are defined in Eqs. (5)-(6) at $z=z_{1}$. $\partial \psi_{m}\left(z_{2} / L\right) / \partial \kappa$ and $\partial \psi_{h}\left(z_{2} / L\right) / \partial \kappa$ can be defined analogous to Eqs. (13) and (14) but replacing $z_{1}$ with $z_{2}, x_{1}$ with $x_{2}$ and $y_{1}$ with $y_{2}$, respectively.

A quasi-Newton method is applied to find the minimum of the cost function $J$. This method requires to perform an iterative procedure to compute the cost function Eq. (1) and its gradients Eq. (9), and to determine the minimum of $\mathbf{J}$ along a search direction via adjusting values of $\kappa$. It is noted that the variational method simultaneously takes into account all information of the wind speed, air temperature and humidity, and that all differences between computed and observed wind, air temperature and specific humidity are minimized simultaneously. Thus, an optimal estimated von Kármán constant can be obtained once the minimization of the cost function is reached. The following iterative procedure is used to obtain the von Kármán constant:

1. Calculate $u, \Delta T$ and $\Delta q$ from Eqs. (2)-(4) with initial values of $u_{*}, T_{*}$ and $q_{*}$;

2. Calculate the cost function gradient (9) with an initial value of $\kappa$, say, $\kappa=0.3$;

3. Perform the iteration to search for a zero of the gradient of the cost function to minimize the cost function;

4. After a new value of $\kappa$ is obtained, repeat Eq. (2)-(4) until the procedure converges.

The convergence criterion is set to $10^{-4}$ and the convergence requirement on searching a zero of the gradient is set to $10^{-7}$. In principle, the weights $W_{u}, W_{T}$ and $W_{q}$ can be expressed to be inversely proportional to their respective observation error variances. In this study, the weights are chosen empirically as $W_{u}=10 \mathrm{~m}^{-2} \mathrm{~s}^{2}, W_{T}=100 \mathrm{~K}^{-2}$ and $W_{q}=1.0 \times 10^{6}$.
Due to the "unclosed" energy balance at the surface (Foken, 2008), a large error ( $>20 \%$ ) may occur in the determination of the latent heat flux from an energy balance equation. In the variational approach, three equations, Eqs. (2)-(4), and two unknown variables, $u_{*}$ and $F_{h}$, form an overdetermined system where $u_{*}$ and $F_{h}$ are solved through the procedure to minimize the difference between observed and computed meteorological variables so that it can adjust the computed flux toward the measured one. Through this process, the observed meteorological and surface conditions are sufficiently taken into account in the variational computation.

Since the Monin-Obukhov similarity theory (MOST) is used as a physical constraint in the iteration procedure, it may not be applicable under some special circumstances. For example, in highly unstable conditions, the shear stress becomes insignificant due to occurrence of local free convection (Garratt, 1992). In highly stable conditions, turbulence tends to be sporadic due to failure of the MOST. In all these cases, even though the iteration in searing the minimum of the cost function Eq. (1) converges, the estimated gradients of wind speed, air temperature and specific humidity can still deviate from observational values due to failure of the Monin-Obukhov similarity relationship. This, in turn, will yield large errors in the retrieved von Kármán constant. To overcome this difficulty, in this study we first impose a constraint $0.35 \leq \kappa \leq 0.45$. The variational-method-computed $\kappa$ values beyond this range will be discarded. Of 6628 data points, over 2700 satisfy this constraint in which about 1900 are in unstable conditions and about 800 in stable conditions. Computational results show that large errors occur mostly in stable conditions. We then relax this constraint in a sensitivity test of the von Kármán constant to atmospheric stability.

\section{Results}

To identify quantitatively the applicability of the von Kármán constant as a scaling factor in the estimate of wind, air temperature and humidity profiles, seven experiments are performed by turning on and off the weights for the wind, air temperature and humidity in the variational formulation, i.e., Eq. (1). The first run, referred to as the control run, considers a realistic state of the atmosphere that includes all information of $u, T$ and $q$. In the next three runs (2-4), only one weight for $u, T$ and $q$ is defined and the rest two weights are taken as zeros. In the last three runs (5-7), two weights of $T$ and $q, u$ and $T$, and $u$ and $q$ are considered and another one is taken as zero. Table 1 lists the weights and variational computed mean values of $\kappa\left(\bar{\kappa}=(1 / n) \sum_{i=1}^{n} \kappa\right)$ under the stable and unstable surface boundary-layer in the different experiments. Value in the bracket of the table indicates the number of samples in each run. In general, $\bar{\kappa}$ is greater in the stable conditions than that in the unstable conditions for all runs. No significant differences among the calculated $\bar{\kappa}$ are found in these runs, especially for stable conditions, as 

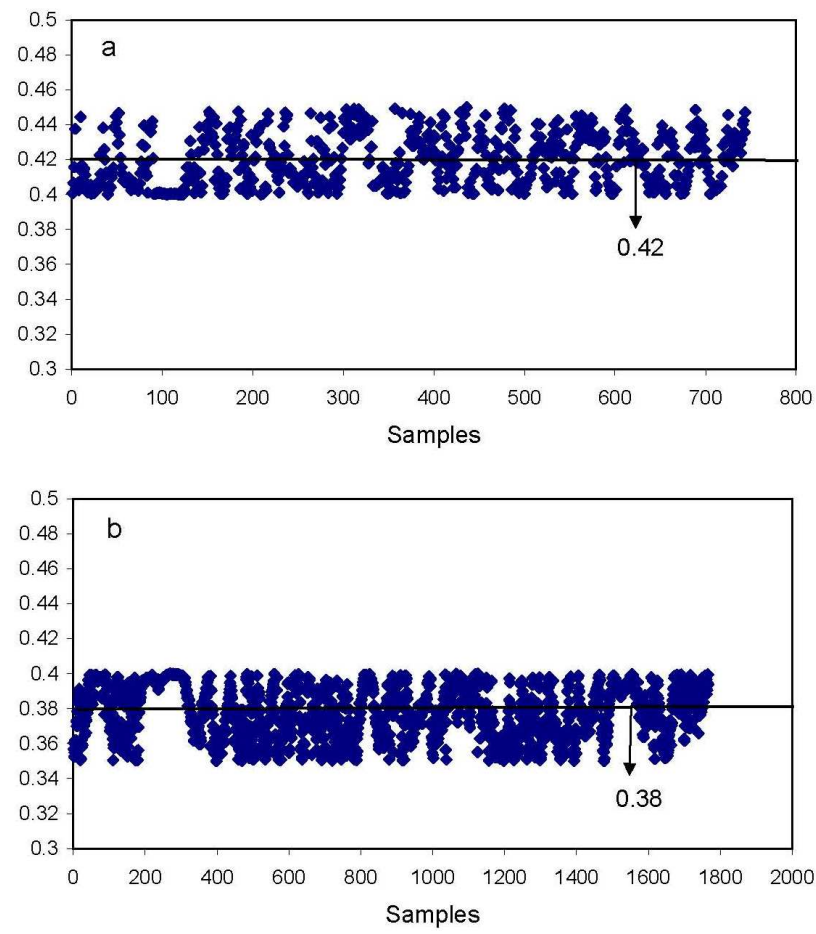

Fig. 1. The von Kármán constant retrieved from the variational analysis under (a) stable conditions, and (b) unstable conditions.

shown in Table 1. This confirms that the scaling factor $\kappa$ can be applied in all logarithmic laws for $u, T$ and $q$.

Figure 1 displays retrieved $\kappa$ values under stable and unstable conditions. As seen, using the Monin-Obukhov relationship described in the last section, the retrieved von Kármán constant appears not a constant anymore. It can be seen from Fig. 2 that $\kappa$ decreases monotonously from the stable to unstable stratification. This decreasing pattern is similar to Andreas et al's results using two different datasets (Andreas et al., 2006). Although $\kappa$ seems to oscillate both in stable and unstable conditions (Fig. 1a and b), our variational computations suggest that $\kappa$ values retrieved from the variational analysis under stable conditions are greater than 0.4 (not averaging), smaller than 0.4 under unstable conditions, and equal to 0.4 under neutral conditions. While all atmospheric stratification is considered, an averaged $\kappa$ value calculated by the variational method with 2594 samples is 0.390. This value is slightly greater than Andreas et al.' value at $0.387 \pm 0.003$ and the same as the $\kappa$ value reported by Frenzen and Vogel (1995). Nevertheless, this value does confirm that the von Kármán constant is smaller than 0.40 , consistent with Andreas et al.'s conclusion (2006).

To test if the retrieved $\kappa$ is sensitive to choices of the profile constants $\left(\gamma_{m}, \gamma_{h}, \beta_{m}, \beta_{h}\right)$, two groups of the profile constants are employed in the variational calculations. The first group of the profile constants, $\left(\gamma_{m}, \gamma_{h}, \beta_{m}, \beta_{h}\right)=(15,9,4.7$, 6.35), was given by Businger et al. (1971) and defined subject

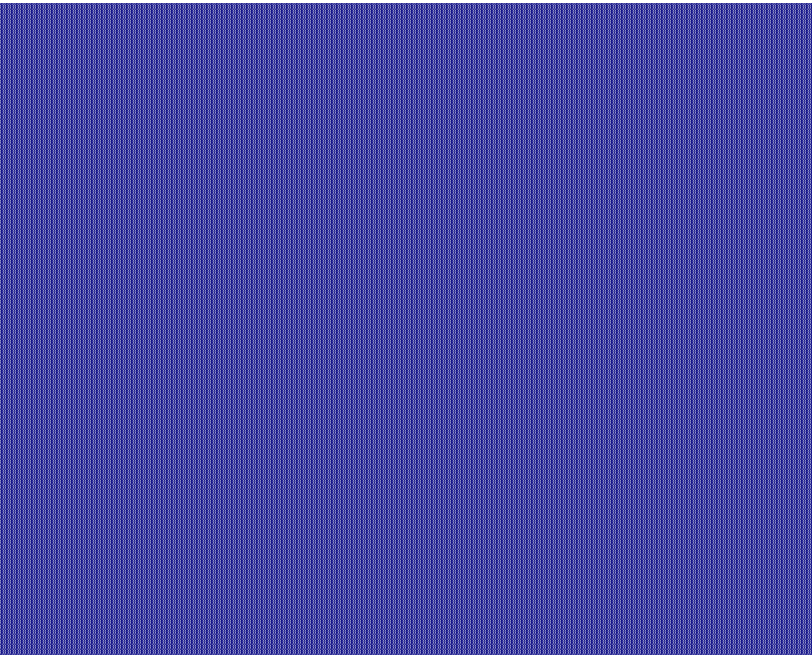

Fig. 2. Variations of $\kappa$ with stability. The profile constants used in the variational computation are $\left(\gamma_{m}, \gamma_{h}, \beta_{m}, \beta_{h}\right)=(15,9,4.7,6.35)$.

to $\kappa=0.35 P_{r t}^{-1}$ ( $P_{r t}$ is Prandtl number and $P_{r t}^{-1}=1.35$ ). Using this set of the profile constants and the CASES-97 dataset, we arrive at an averaged $\kappa$ value at 0.390 (2954 samples), as stated above. As shown in Fig. 2, in neutral conditions $\kappa$ is equal to 0.40 . The second group of the profile constants, $\left(\gamma_{m}, \gamma_{h}, \beta_{m}, \beta_{h}\right)=(19.3,11.6,6,7.8)$, was defined subject to $\kappa=0.40$ and $P_{r t}^{-1}=1.05$ (Högström, 1988; Foken, $2006,2008)$. Using this group of constants the variational analysis yields an averaged $\kappa$ value at 0.389 (2811 samples), which is almost the same as Andreas et al.'s value (2006) but slightly lower than $\kappa$ value (0.390) obtained using Businger et al.'s profile constants. In calculations, we did not predefine a $\kappa$ value following either Businger et al. (1971) or Högström (1988) work but just gave randomly an "initial $\kappa$ value" (say, $\kappa=0.3$ ) in the iteration procedure of the variational method. Again we find that in neutral conditions $\kappa$ is equal to 0.40 , computed using the second group of the profile constants. This result appears to suggest that the determination of the profile constants seems not to depend on the von Kármán constant. If we relax the constraint $0.35 \leq \kappa \leq 0.45$ imposed to the variational computed results but simply introduce a new condition which only rejects calculated $\kappa$ values greater than 0.8 , we then found that under unstable conditions $\kappa=0.340$ using the first group of profile constants and 0.351 using the second group of constants. For the stable cases, $\kappa=0.493$ using the first group of profile constants and 0.490 using the second group of constants. Overall, using the classical Monin-Obukhov similarity relationship (Businger - Dyer profile relation) and those profile constants the von Kármán constant in the non-neutral stratification deviates from its value (0.4) in the neutral stratification. This agrees with Andreas et al's results, obtained using SHEBA (Surface Heat Budget of the Arctic Ocean) dataset (2006), showing 


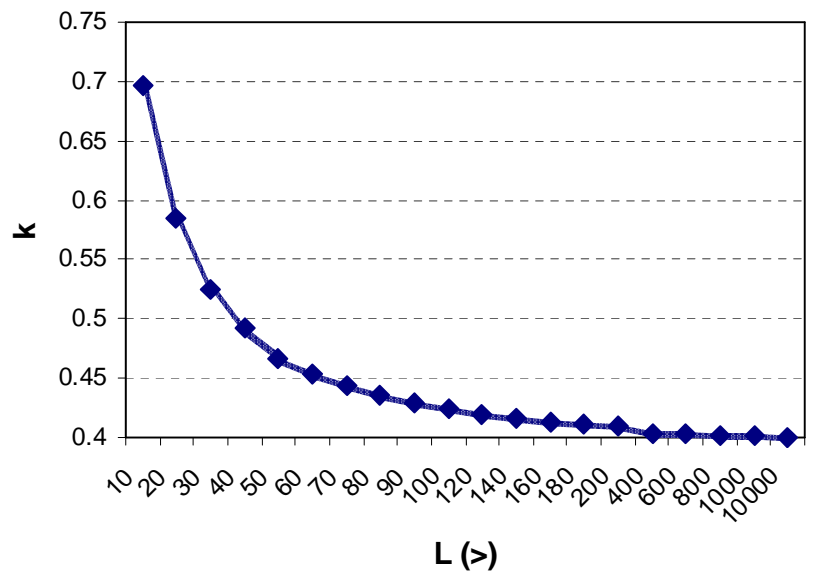

Fig. 3. Change in the von Kármán constant with the Obukhov length under stable surface boundary-layer. Results are obtained by imposing the condition $10<L<10000$.

dependence of the von Kármán constant on stratification. Following Andreas et al., a stratification correction is made to the variational calculated $\kappa$ values. This yields a mean value of the von Kármán constant at 0.401 . Hence, using the constant $\kappa$ value at 0.4 in all atmospheric stability without a stratification correction will lead to errors in the estimate of wind speed and scalars profiles, as well as surface fluxes under the non-neutral conditions. These errors would increase with the increase in the strength of atmospheric stability, as shown in Fig. 2.

Additional computations were conducted to test the sensitivity of the von Kármán constant to atmospheric stability by relaxing the imposed constraint $0.35 \leq \kappa \leq 0.45$. Instead, we simply impose a condition which limits $\kappa \leq 0.6$. This yields $\kappa$ values at 0.428 for stable condition and 0.340 for unstable condition. The mean value of the von Kármán constant with total 3563 samples is 0.384 under all atmospheric stability conditions. If we further impose the condition $\kappa \leq 0.7$ in the variational calculation, we obtain $\kappa=0.453$ for stable conditions. The number of samples satisfying the condition for the stable atmosphere $(\kappa \leq 0.7)$ increases from 778 (for $\kappa \leq 0.6$ ) to 859. This suggests greater uncertainties in determination of the von Kármán constant in the stable boundary-layer compared with unstable conditions. It is important to indicate that, because the majority of the stable cases are rejected by the restrictions on $\kappa$ in our calculations, the mean $\kappa$ value of 0.384 is, in reality, weighted to unstable conditions.

Instead of imposing conditions on the von Kármán constant, we now introduce constraints subject to stable stratifications by setting the Obukhov length $L>10,20, \ldots, 10000$. Figure 3 illustrates the change in the von Kármán constant with these conditions in the stable boundary-layer using Högström's universal constants (1988; Foken, 2006). As shown, the von Kármán constant tends asymptomatically to
0.4 from very stable to neutral condition $(L \rightarrow \infty)$. The figure also shows that, if we use the restriction $0.35 \leq \kappa \leq 0.45$ in the variational computation, $\kappa$ values under the stable atmosphere with $L<60$ would be rejected by this constraint. This suggests that the von Kármán constant cannot be determined precisely in the very stable atmospheric boundary-layer.

A question might be raised for the significance of the difference between $\kappa$ values at 0.39 and 0.4 when the measurement errors are taken into account. As aforementioned, our calculated values with and without the constraint for $\kappa$ ranges from 0.384 to 0.390 , which is within the error range of Andreas et al.'s (2006) value at $0.387 \pm 0.003$. In the present study, the measurement errors were taken into account by introducing the dimensional weights for wind, air temperature and humidity profiles in the cost function (Eq. 1), defined to be inversely proportional to their respective observation error variances. This is one of advantages of the variational technique. The statistical difference between variational computed $\kappa$ values and the $\kappa$ value at 0.4 has been determined using a t-test. Based on the calculation, the statistic $t$ under the null hypothesis $H_{o}$, is equal to $6.27\left(>t_{0.005 / 2}=2.6\right)$ with the statistically significant level of $99.5 \%$. This suggests that the statistical difference between $\kappa$ values at 0.39 and 0.4 is significant. In addition, it is not clear if the variational calculated von Kármán constant would be sensitive to wind, air temperature and humidity profiles at different observation levels, because these profiles are not linear in the vertical. The CASES-97 dataset provided only air temperature and humidity at two levels above the ground surface. The ratio of the measuring heights $(2$ and $1 \mathrm{~m})$ is likely not higher enough to determine gradients in the surface layer with a high accuracy (Foken, 2008). The lower level at $1 \mathrm{~m}$ could be also influenced by the roughness sublayer. A remedy is to use multiple level measurement data. Further study of this aspect is needed by using multiple level observations. There are likely other factors that affect the determination of the von Kármán constant. In the present study, the physical constraints in the cost function (Eq. 1) are expressed by the Monin-Obukhov similarity relationships from which the von Kármán constant is retrieved. Therefore, uncertainties in the MOST in estimation of profiles of wind, air temperature and humidity as well as momentum and heat fluxes, especially under very stable and strong convection conditions, would inevitably yield errors in the evaluation of the von Kármán constant. On the other hand, given that the advantage of the variational method is that it is able to fully take into account information of the existing MOST and measured meteorological conditions over an underlying surface, the variational method has led to substantial improvements over the conventional MOST-based flux-gradient method (Cao and Ma, 2005; Cao et al., 2006). We would expect that the variational estimated $\kappa$ values would be more accurate than that derived from the conventional method.

It has been arguable that the von Kármán constant might depend on the roughness Reynolds number (e.g., Frenzen 

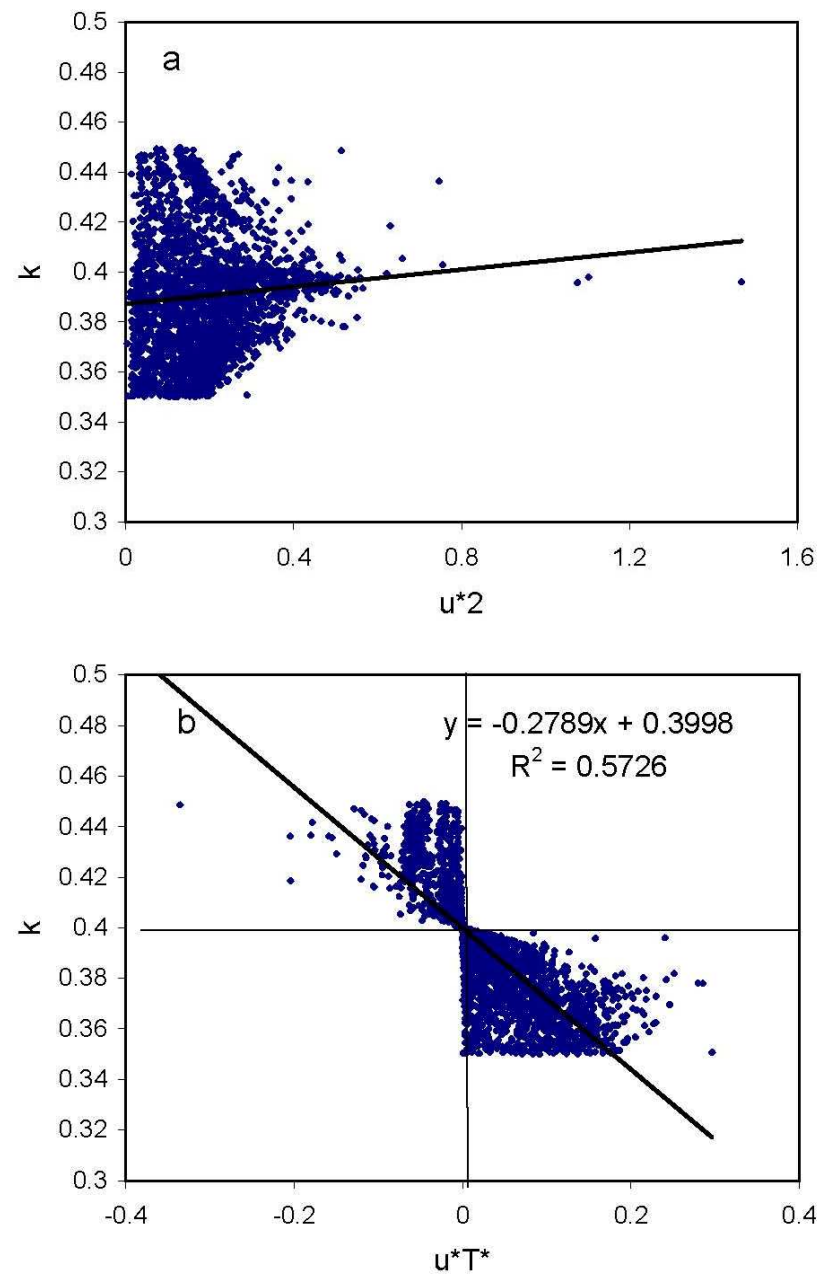

Fig. 4. Variation of $\kappa$ with (a) momentum flux $u_{*}^{2}\left(\mathrm{~m}^{2} \mathrm{~s}^{-2}\right)$ and (b) heat flux $u_{*} T_{*}\left(\mathrm{~m} \mathrm{~s}^{-1} \mathrm{~K}\right)$. Linear equations of fitting line are also displayed. $R^{2}$ is the coefficient of determination.

and Vogel, 1995; Andreas et al., 2006). In the present study, the roughness length was neither used in the model nor retrieved from the variational algorithm. Thence, we did not attempt to evaluate the relationship between the von Kármán constant and the roughness Reynolds number. Instead, we illustrate retrieved values of the von Kármán constant against the momentum flux $u_{*}^{2}$ (surface shear stress) because the friction velocity is proportional to the roughness Reynolds number. As shown in Fig. 4a, $\kappa$ appears to increase slightly with the increase of $\mathrm{u}_{*}^{2}$, as seen from the fitting line but there is large deviation of $\kappa(<0.4$ and bellow the fitting line) from the fitting line. This indicates that the von Kármán constant does not change significantly with $u_{*}^{2}$. The relationship between the von Kármán constant and heat flux $\left(u_{*} T_{*}\right)$ is displayed in Fig. 4b. As shown, $\kappa$ is greater than 0.4 when the heat fluxes are negative (downward flux) and smaller than 0.4 when the $u_{*} T_{*}$ are positive (upward flux). The results

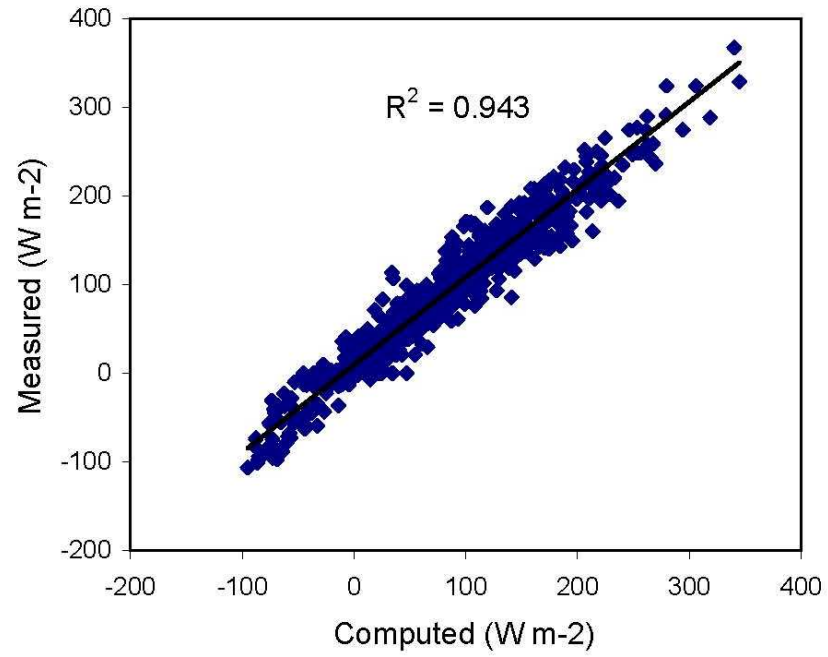

Fig. 5. Correlation diagram for variational calculated and measured heat flux.

presented in Fig. 4b are, in fact, consistent with Fig. 2 and the finding described before. Namely, $\kappa$ is greater than 0.4 under stable conditions and smaller than 0.4 under unstable conditions. For the neutral stability $u_{*} T_{*}=0, \kappa$ is equal to 0.4 . This confirms merely the variation of $\kappa$ with stability as shown in Figs. 2 and 3. There appears no clear functional relationships between $\kappa$ and $u_{*} T_{*}$.

To test the accuracy of the variational analysis applied in this study, we compare the computed and measured sensible heat fluxes. The computed sensible heat fluxes is defined by $H=-c_{p} \rho u_{*} T_{*}$, where $\rho$ is the air density, $c_{p}$ is the specific heat of air at constant pressure, $u_{*}$ and $T_{*}$ are derived from the variational computation, respectively, as described in Sect. 3. The results are plotted in Fig. 5. As seen, the computed sensible heat fluxes agree very well with the measured heat fluxes (note that a constraint $0.35 \leq \kappa \leq 0.45$ is imposed in the analysis). These agreements are at least due partly to adjusting $\kappa$ value in the variational computation that helps to minimize the errors between the estimated and measured fluxes. Table 2 lists the RMS errors and relative errors between the estimated and measured gradients of the wind speed, air temperature, specific humidity and heat fluxes. The RMS error is expressed as $E_{r}=\left(\sum_{i=1}^{n} s_{i}^{2} / n\right)^{1 / 2}$, where $s_{i}$ is the difference between the estimated and measured quantities. For example, the difference between estimated and measured wind speed gradient is $s_{i}=\Delta u_{i}-\Delta u_{i}^{\text {obs }}$. The relative error for wind speed gradient can be written as

$$
R e=\left(\sum_{i=1}^{n}\left(\Delta u_{i}-\Delta u_{i}^{\mathrm{obs}}\right)^{2} / \sum_{i=1}^{n} \Delta u_{i}^{2}\right)^{1 / 2} .
$$


Table 2. RMS and relative errors between estimated and measured gradients of wind speed, temperature, specific humidity and sensible heat flux.

\begin{tabular}{cccccccc}
\hline \multicolumn{2}{c}{$\Delta u\left(\mathrm{~m} \mathrm{~s}^{-1}\right)$} & \multicolumn{2}{c}{$\Delta T(\mathrm{~K})$} & \multicolumn{2}{c}{$\Delta q$} & \multicolumn{2}{c}{$H\left(\mathrm{~W} \mathrm{~m}^{-2}\right)$} \\
RMS & RE & RMS & RE & RMS & RE & RMS & RE \\
\hline $0.7169 \mathrm{E}-2$ & $0.9360 \mathrm{E}-2$ & $0.1305 \mathrm{E}-2$ & $0.4883 \mathrm{E}-2$ & $0.4004 \mathrm{E}-6$ & $0.5435 \mathrm{E}-2$ & 7.2334 & 0.2010 \\
\hline
\end{tabular}

Clearly, both RMS and relative errors between the estimated and measured gradients of wind speed, air temperature and specific humidity are very small. For the sensible heat flux, RMS and RE are equal to 7.2 and $0.2 \mathrm{~W} \mathrm{~m}^{-2}$, respectively.

\section{Concluding remarks}

A variational method was developed to retrieve the von Kármán constant. This method made use of full information of measured gradients of the wind speed, air temperature and humidity. It was found from the variational analysis using over 2700 samples collected in CASES-97 (Surface Qualls Surface Site) field campaign that $\kappa$ is a constant at a value of 0.40 for neutral stratification. For non-neutral stratification, the von Kármán constant is greater than 0.4 under stable conditions and smaller than 0.4 under unstable conditions. When all atmospheric stratifications were taken into account, the averaged $\kappa$ value is $0.384-0.390$. Using the classical Monin-Obukhov similarity relationship associated with different profile constants, overall results show that $\kappa$ decreases monotonously from stable to unstable stratification. It is anticipated that for very stable conditions, the von Kármán constant becomes indeterminate. Deviation of $\kappa$ from its constant value (0.4) in the neutral stratification becomes significant as strong stable and unstable stratification take place. Therefore, a constant value of $\kappa$ at $0 . .40$ in all atmospheric stratifications without stability correction is likely to yield errors in the estimate of the profiles of wind speed and scalars, as well as surface fluxes under the non-neutral conditions. Our results also confirm Högström (1996) and Andreas et al. (2006)'s finding that, for a given von-Kármánconstant at 0.40 is, the universal functions may yield a deviation of $10-20 \%$ from $\kappa=0.40$.

Attention should therefore be paid for the hypothesis of the constant $\kappa$ in the non-neutral atmospheric stratification. A reevaluation of these relationships (Eqs. 2-7a, b), or at least those profile constants (in Eqs. 6a, b-7a, b), might become necessary. As Frenzen and Vogel (1995) noticed that, in the form of most often used Monin-Obukhov similarity relationships, namely, the Businger-Dyer relation $\phi_{m}=\left(1-\gamma_{m} z / L\right)^{-1 / 4}$, decreasing $\kappa$ by $5 \%$ increases $\gamma_{m}$ by more than $60 \%$. Currently, it is not clear if the changes in the profile constants could help to reduce the deviation of $\kappa$ in the non-neutral stratification from its constant value at 0.4 under the neutral stratification. Our variational calculation suggested that the von Kármán constant appeared not to respond strongly to the profile constants.

Acknowledgements. The surface flux, meteorological and soil observations (Qualls Site) were collected and analyzed by Russell J. Qualls at the University of Colorado, Boulder, Colorado.

Edited by: J. Rinne

\section{References}

Andreas, E., Claffey, K. J., Jordan, R. E., Fairall, C. W., Guest, P. S., Persson, P. O., and Grachev, A. A.: Evaluations of the von Kármán constant in the atmospheric surface layer, J. Fluid Mach., 559, 117-149, doi:10.1017/S0022112006000164, 2006.

Businger, J. A., Wyngaard, J. C., Izumi, Y., and Bradley, E. F.: Flux profile relationships in the atmospheric surface layer, J. Atmos. Sci., 28, 181-189, 1971.

Cai, X. M. and Steyn, D. G.: The von Kármán constant determined by large eddy simulation, Bound.-Lay. Meteorol., 78, 143-164, 1996.

Cao, Z. and Ma, J.: An application of the variational method to computation of sensible heat flux over a deciduous forest, J. Appl. Meteorol., 44, 144-152, 2005.

Cao, Z., Ma, J., and Wayne, R. R.: Improving computation of sensible heat flux over a water surface using the variational method, J. Hydrometeorol., 7, 678-686, 2006.

Fleagle, R. G. and Businger, J. A.: An Introduction to Atmospheric Physics, 2dn Ed., Academic Press, New York, 1980.

Foken, T.: 50 years of the Monin-Obukhov similarity theory, Bound.-Lay. Meteorol., 119, 431-447, 2006.

Foken, T.: Micrometeorology. Springer, Berlin, Heidelberg, 2008.

Frenzen, P. and Vogel, C. A.: On the magnitude and apparent range of variation of the von Karman constant in the atmospheric surface layer, Bound.-Lay. Meteorol., 72, 371-392, 1995.

Garratt, J. R.: The Atmospheric Boundary Layer, Cambridge University Press, New York, 1992.

Högström, U.: Non-dimensional wind and temperature profiles in the atmospheric surface layer: A re-evaluation, Bound.-Lay. Meteorol., 42, 55-78, 1988.

Högström, U.: Review of some basic characteristics of the atmospheric surface layer, Bound.-Lay. Meteorol., 78, 215-246, 1996.

Kondo, J. and Sato, T.: The Determination of the von Karman constant, J. Meteorol. Soc. Japan, 60, 461-470, 1982. 
Ma, J. and Daggupaty, S. M.: Using all observed information in a variational approach in measuring $z_{0 m}$ and $z_{0 t}$, J. Appl. Meteorol., 39, 1391-1401, 1999.

Parlange, M. B. and Katul, G. G.: Watershed scale stress from Tethersonde wind profile measurements under near neutral and unstable atmospheric stability, Water Resour. Res., 31, 961-968, 1995.

Sheppard, P. A.: The aerodynamic drag of the Earth's surface and the value of Karman's constant in the lower atmosphere, Proc. Roy. Soc. London Ser. A, 188, 208-222, 1947.
Yaglom, A. M.: Comments on wind and temperature flux-profile relationships, Bound.-Lay. Meteorol., 11, 89-102, 1977.

Zhang, S. F., Oncley, S. P., and Businger, J. A.: Evaluation of the von Karman constant from a new surface layer experiment, Proc, in: Eighth Symp. On Turbulence and Diffusion, San Diego, CA, 25-29 April, Am. Meteorol. Soc., 148-150, 1988. 